Anales de Geografía de la Universidad Complutense ISSN: 0211-9803

http://dx.doi.org/10.5209/rev_AGUC.2016.v36.n1.52715

\title{
Reorganización administrativa territorial como estrategia para optimizar la estructura de la Administración local
}

\author{
Tomás Llorente Aguado ${ }^{1}$; Jesús Molina Saorín ${ }^{2}$ \\ Recibido: 23 de septiembre del 2015 / Enviado a evaluar: 23 de enero del 2016 / Aceptado: 3 de mayo del 2016
}

Resumen. Durante la durísima experiencia económica, social y de falta de valores que se ha atravesado en los últimos años, las Administraciones públicas -especialmente en Grecia, Portugal, Italia y Españase han visto obligadas a reconsiderar fuertemente la estructura y el tamaño de su sector público. A pesar de algunas iniciativas encaminadas a reducir el número de municipios y provincias, pocos avances sustanciales se han producido en la mejora de la gestión de la Administración pública local durante esta crisis. En este estudio se propone la reorganización administrativa territorial como estrategia para optimizar la estructura de la Administración local, analizando la situación española -en general- y de una comunidad autónoma en particular.

Palabras clave: Administración pública; eficiencia; ordenación territorial; competencias públicas.

\section{[en] Territorial administrative reorganization as a strategy for optimizing the structure of local government}

\begin{abstract}
Through the tough economic and social situation and the lack of values that has been experienced in recent years, local governments especially in Greece, Portugal, Italy and Spain have been forced to strongly consider the structure and size of their public sector. Despite some initiative to reduce the number of municipalities and provinces, little substantive progress has been made in improving the management of the local public Administration during this crisis. In this study a territorial administrative reorganization is proposed as a strategy to optimize the structure of local government, analyzing the Spanish situation in general, and an autonomous community in particular.
\end{abstract}

Keywords: Public Administration; efficiency; territory organization; public competences.

1 Ayuntamiento de Abarán (Murcia)

E-mail: 14555llorente@coam.es

2 Universidad de Murcia

E-mail: jesusmol@um.es 


\section{[fr] Réorganisation administrative territoriale comme une stratégie d'optimisation de la structure de l'administration locale}

Résumé. Au cours de cette situation économique et sociale difficile et l'absence de valeurs qui a été connu au cours des dernières années, les gouvernements locaux en particulier en Grèce, au Portugal, en Italie et en Espagne ont été contraints d'envisager sérieusement la structure et la taille de leur secteur public. Malgré une certaine initiative visant à réduire le nombre de municipalités et les provinces, peu de progrès substantiels ont été accomplis dans l'amélioration de la gestion de l'administration publique locale au cours de cette crise. Dans cette étude, une réorganisation administrative territoriale est proposée comme une stratégie visant à optimiser la structure de l'administration locale. On analyse la situation espagnole en général, et une communauté autonome en particulier.

Mots clés: L'administration publique; l'efficacité; l'aménagement du territoire; les pouvoirs publics.

Cómo citar. Llorente Aguado, T. y Molina Saorín, J. (2016): Reorganización administrativa territorial como estrategia para optimizar la estructura de la Administración local. Anales de Geografía de la Universidad Complutense, 36(1), 91-108.

Sumario. 1. Introducción. 2. Distribución de municipios en España. 3. Municipio frente a provincia, ¿dicotomía o complementariedad en la Administración local? 3.1. Determinación de las competencias en las provincias españolas. 3.2. Agrupación municipal. 4. Intervención de las diputaciones. 5. Asociaciones municipales. 6. Particularización de las medidas y propuestas de aplicación del Método DEA (Data Envelopment Anallysis). 7. Conclusiones. 8. Bibliografía.

\section{Introducción}

La Administración pública se rige por una legislación heredada del siglo XIX y muestra de ello es que un inmenso número de poblaciones actuales provienen de la Constitución de 1812, que obligaba a disponer de ayuntamiento en los pueblos en los que residan 1.000 almas, incluyendo los residentes en el casco urbano y en el resto del territorio que lo forma. Con el fin de reducir el gasto público y facilitar la gestión de los servicios en la Administración (especialmente local), existen varias líneas de actuación previstas en la legislación vigente, y aplicadas por toda la geografía nacional. Estas propuestas -y las prácticas llevadas a cabo por las diversas Administraciones- varían desde la agrupación municipal hasta la gestión de servicios por parte de entes supramunicipales (como las mancomunidades o consorcios), llegando algunos municipios a delegar -incluso- varias de sus competencias en otras administraciones como las Diputaciones Provinciales. Algunas de estas prácticas han sido aplicadas desde mediados del pasado siglo en ciertos países de Europa (Feria y Andújar, 2015), no sin antes haber definido claramente las competencias de cada nivel administrativo, a diferencia de la indeterminación predominante en España. En algunos Estados miembros de la Unión Europea, y especialmente en aquellos que están en proceso de adhesión, puede apreciarse una tendencia general a considerar que el mayor número posible de servicios deben ser prestados por el nivel de gobierno local. A pesar de esta tendencia, en el entorno español ha predominado la estrategia del sur de Europa (Rodríguez, 2009), que ha sido seguida en Francia, Italia y -a partir de la década de 1990 - también en Grecia. Esta estrategia ha intentado mantener el histórico formato de municipios pequeños con la creación de estructuras 
intermunicipales, que asisten y apoyan a los municipios en el ejercicio de sus competencias. Está basada en el principio de la cooperación voluntaria y, por tanto, en la máxima de que las fusiones únicamente pueden llevarse a cabo con el consentimiento de los municipios o de sus habitantes. Esta intención de mantener municipios de pequeñas dimensiones ha llevado a la necesidad de crear un amplio espectro de entidades intermunicipales para dar apoyo a estos municipios (Bosque y García, 2000; Pérez y Gutiérrez, 2013), hecho que ha derivado en solapamientos e ineficiencias, ralentizando la toma de decisiones, generando conflictos y rivalidades, e incrementando los costes de transacción y conflicto. En general, los gobiernos locales en el sur de Europa gozan de menos competencias de este tipo que los municipios ubicados en el norte del continente. Esto significa que la Administración Pública cercana se concentra en el nivel local de gobierno, mientras que el nivel federal (o nacional) se ocupa de la denominada alta política (relaciones internacionales y seguridad), junto con la regulación del estado de bienestar, siendo los niveles regionales o estatales quienes tienen asignadas las competencias en materia de educación (incluyendo la educación superior), la seguridad interior (Policía) y el desarrollo económico de sus territorios. Idéntico escenario es aplicable al Reino Unido, así como a Dinamarca, Noruega y Suecia, donde -tradicionalmente- el nivel local de gobierno ejerce las competencias más relevantes para el día a día de los ciudadanos. En algunos de estos países, el gobierno local tiene incluso que autofinanciarse, a través de impuestos y tasas locales con los que hacer frente a costosos gastos derivados de ese estado de bienestar, cuyo núcleo históricamente se ha considerado que ha de estar en manos de los gobiernos locales como una competencia originaria (Jiménez, 2012).

\section{Distribución de municipios en España}

En la actualidad existen más de 8.120 municipios en España, de los que 2.627 son poblados por menos de 250 habitantes, y 2.235 por menos de 1.000 . Si observamos el número de municipios inferior a 5000 habitantes, obtenemos un total de 6.809, lo que supone un $84 \%$ del total. Por el contrario, únicamente existen seis municipios de más de 500.000 habitantes. Tales municipios -Madrid, Barcelona, Valencia, Sevilla, Zaragoza y Málaga- aglutinan al 16,28\% de la población española en tan solo un $0,48 \%$ de la superficie nacional. Se puede entender claramente que nos encontramos ante una distribución desigual en lo que corresponde a la relación de población respecto de la superficie que ocupan. Las características de superficie y población de los municipios varían -significativamente- de una región a otra, encontrando notables diferencias en el territorio nacional. Para conocer cómo se estructura la distribución de los municipios en España, se han elaborado las siguientes tablas y figuras; precisamente en la imagen siguiente (Figura 1 ) se pueden apreciar las zonas donde se encuentran los municipios más dispersos en el territorio, frente a las zonas de mayor concentración. 
Figura 1. Identificación de zonas de alta y baja concentración de municipios por comunidad autónoma.

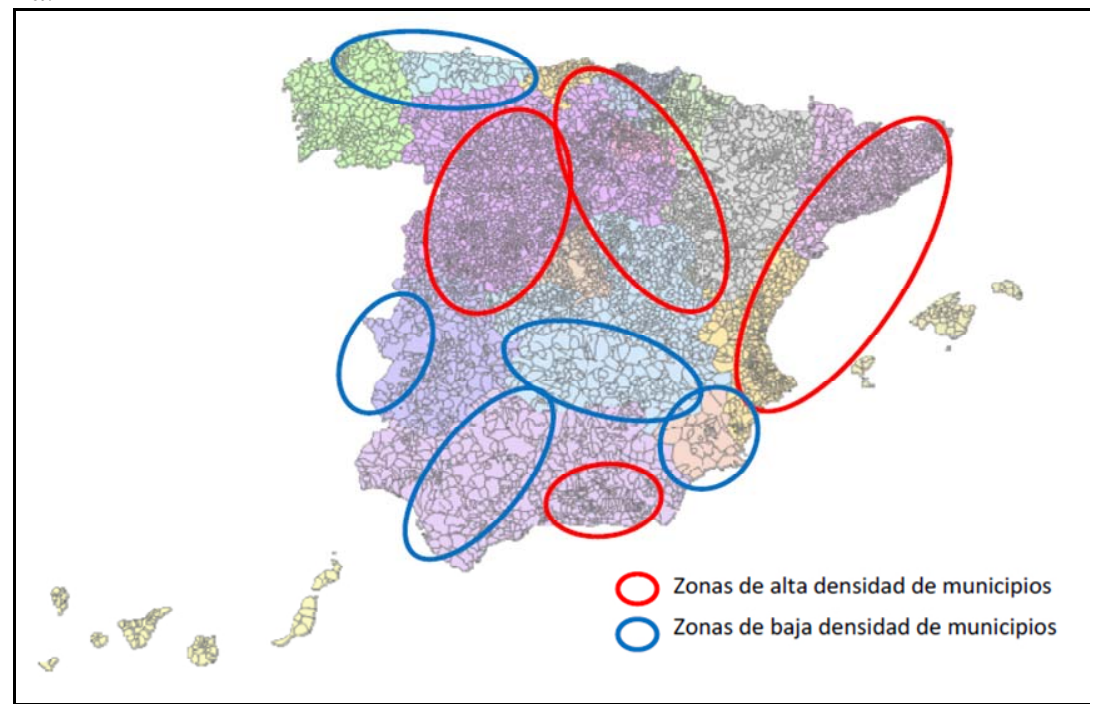

Fuente: INE, 2012.

Tal y como puede apreciarse (Figura 1), se observan las zonas con mayor y menor número de municipios en relación a la superficie; es posible apreciar una primera aproximación sobre la predisposición inicial en la organización territorial de cada provincia -en general-. A la cabeza en cuanto al mayor número de municipios por provincia, encontramos Salamanca, Burgos y Barcelona; con menos municipios por provincia aparecen -como posteriormente se recoge- Asturias, Lugo, Cádiz, Sevilla, Jaén, Albacete y Murcia.

A la luz de los datos anteriores (Tabla 1), cabe destacar la gran diferencia en la media de superficie por municipio en las provincias de Vizcaya y Murcia, teniendo esta última una media de superficie de sus municipios 12,7 veces superior a la de la primera. El resultado de comparar la media de las diez provincias con mayor y menor media de superficie por municipio arroja una proporción 5,6 veces superior de las diez primeras con respecto a las diez últimas. Esta razón permite considerar que los criterios a la hora de emprender iniciativas en la dirección de agrupar municipios no podrán -a priori- seguir los mismos patrones en todo el territorio nacional. Partiendo de esa base, se extraen varias conclusiones del cuadro anterior. Si observamos simplemente el número de municipios por provincia, vemos que la relación entre los grupos de provincias analizadas es de 2,5 a 1 . Por el contrario, si comparamos la media de superficie de estas provincias la relación es de 1 a 2 . Es decir, las diez provincias que menos densidad de municipios albergan, tienen 2,5 veces menos de municipios en el doble de superficie, que aquellas diez provincias con más densidad de municipios. 
Tabla 1. Valores demográficos asociados al número de municipios de las provincias con menor y mayor densidad de municipios en España.

\begin{tabular}{|c|c|c|c|c|c|c|}
\hline Provincia & $\begin{array}{c}\mathrm{N}^{\circ} \\
\text { Municipios } \\
\end{array}$ & Extensión & $\begin{array}{c}\text { Superficie/N. } \\
\text { municipios }\end{array}$ & Población & $\begin{array}{c}\text { Población/N. } \\
\text { municipios }\end{array}$ & $\begin{array}{c}\quad \mathrm{N.}^{\circ} \\
\text { Municipios } \\
\text { /Superficie }\end{array}$ \\
\hline Murcia & 45 & 11.317 & 251,49 & 1.461 .979 & 32.488 & 0,4 \\
\hline Ciudad Real & 102 & 19.749 & 193,62 & 529.453 & 5.191 & 0,52 \\
\hline Córdoba & 75 & 13.718 & 182,91 & 805.108 & 10.735 & 0,55 \\
\hline Albacete & 87 & 14.858 & 170,78 & 401.682 & 4.617 & 0,59 \\
\hline Cádiz & 44 & 7.385 & 167,84 & 1.236 .739 & 28.108 & 0,6 \\
\hline Lugo & 67 & 9.803 & 146,31 & 353.504 & 5.276 & 0,68 \\
\hline Jaén & 97 & 13.498 & 139,15 & 670.761 & 6.915 & 0,72 \\
\hline Asturias & 78 & 10.565 & 135,45 & 1.084 .341 & 13.902 & 0,74 \\
\hline Sevilla & 105 & 14.001 & 133,34 & 1.917 .097 & 18.258 & 0,75 \\
\hline Badajoz & 164 & 21.657 & 132,05 & 692.137 & 4.220 & 0,76 \\
\hline Vizcaya & 112 & 2.217 & 19,79 & 1.153 .724 & 10.301 & 5,05 \\
\hline Guipúzcoa & 88 & 1.997 & 22,69 & 707.263 & 8.037 & 4,41 \\
\hline Barcelona & 311 & 7.733 & 24,86 & 5.511 .147 & 17.721 & 4,02 \\
\hline Gerona & 221 & 5.886 & 26,63 & 753.046 & 3.407 & 3,75 \\
\hline La Rioja & 174 & 5.034 & 28,93 & 322.415 & 1.853 & 3,46 \\
\hline Ávila & 248 & 8.048 & 32,45 & 171.896 & 693 & 3,08 \\
\hline Segovia & 209 & 6.949 & 33,25 & 164.268 & 786 & 3,01 \\
\hline Salamanca & 362 & 12.336 & 34,08 & 353.619 & 977 & 2,93 \\
\hline Tarragona & 183 & 6.283 & 34,33 & 808.420 & 4.418 & 2,91 \\
\hline Valladolid & 225 & 8.202 & 36,45 & 533.640 & 2.372 & 2,74 \\
\hline
\end{tabular}

Fuente: Elaboración propia. (Leyenda: Valores mínimos /Valores máximos).

Ahondando un poco más en los datos ofrecidos en la tabla anterior, se desprende que las dos provincias formadas por menos municipios -Cádiz y Murcia- tienen las mayores poblaciones de su serie, ofreciendo una población media por municipio superior -incluso- a la provincia de Barcelona, a pesar de contar con 5,5 millones de habitantes. Si analizamos la densidad de población de las dos series, observamos que la serie de provincias con más municipios por superficie están muy influenciadas por Barcelona, Vizcaya y Guipúzcoa. No obstante, una vez hallada la densidad de población de las series en cada caso ( $\sin$ las tres primeras provincias), se aprecia que 
las provincias con mayor densidad de municipios continúan siendo más densas -por superficie- que las provincias con menos municipios, de lo que se desprende que si se agruparan los municipios de las primeras, la (posible) desertificación de los nuevos municipios agrupados no supondría -a priori- problema alguno; tampoco la mayor extensión de los municipios resultantes de la agrupación supondría una dificultad desconocida en España, ya que las provincias con menor densidad de municipios arrojan una media de superficie/número de municipios 5,5 veces mayor.

\section{Municipio frente a provincia, ¿dicotomía o complementariedad en la administración local?}

\subsection{Determinación de las competencias en las provincias españolas}

El origen de la regulación de la Administración Local proviene de la Constitución promulgada en Cádiz (1812), y se afianzó durante el Trienio Liberal (1820-1823); aquella legislación se caracterizó por la implantación de ayuntamientos en cada población que contase con -al menos- 1.000 habitantes, y por el tendido de la red provincial en torno al binomio Diputación-Jefe político. Los integrantes de los ayuntamientos serían elegidos por sufragio indirecto. La influencia de la incipiente revolución de septiembre al régimen local -que se concretó en la legislación municipal y provincial de 1870- supuso la adopción de sufragio universal en la elección de todos los cargos municipales, con el consiguiente robustecimiento de las diputaciones provinciales. En el plano provincial, las diputaciones han permanecido subordinadas -por completo- a los gobernadores civiles; en el municipal, los ayuntamientos (escasamente representativos) continúan sometidos a la estrecha tutela del Estado. En ese sentido, el poder central se encuentra investido de sobradas atribuciones para intervenir en la designación de los alcaldes, remover a las autoridades locales o suspender los acuerdos municipales. Los criterios a que respondía la legislación local mencionada, lejos de infundir vitalidad a ayuntamientos y diputaciones, propiciaron su parálisis. El proyecto de Maura (1907), fue el más serio y meditado intento de reconsideración del régimen local -en su conjunto-, de lucha contra la corrupción y en favor del reforzamiento de los organismos municipales y provinciales (Parejo, 2007). Dicho proyecto reconocía la diversidad local, derogaba las disposiciones desamortizadoras, fortalecía la posición de los alcaldes, relajaba la tutela del Estado y -simultáneamente- pretendía extender la acción de los entes locales por la vía -entre otras- de la municipalización de servicios (tal y como recoge el preámbulo de la Ley 7/1985 Reguladora de las Bases del Régimen Local). Por su parte, el Real Decreto Legislativo 781/1986 (de 18 de abril), por el que se aprueba el Texto Refundido de las Disposiciones Legales vigentes en materia de Régimen Local, en su Título III regulaba la Provincia del siguiente modo:

- La creación, modificación y disolución de organismos y establecimientos provinciales. 
- Informar en los expedientes de fusión, agregación o segregación de municipios de su territorio.

- La provincialización de servicios.

En la actualidad, las diputaciones habrán de contribuir-dentro de sus competencias- al cumplimiento de los servicios públicos impuestos a los municipios. Esta cooperación podrá ser total o parcial, según aconsejen las circunstancias económicas de los municipios interesados. Qué duda cabe, la composición y organización de las provincias en España resulta llamativa, pues los diputados no son electos por el pueblo, lo que puede suponer un problema de legitimidad ya que una de las competencias de la provincia es coordinar fondos para los diversos municipios (Heilnet, 2010). Las diputaciones provinciales españolas tienen una de las más bajas capacidades político-administrativas en comparación con el resto de Europa, y las acciones que realizan no siempre aportan valor a los ciudadanos (Reguera, 2012). Las diputaciones provinciales españolas tienen reconocidas - de manera muy limitada- sus competencias originarias. Queda patente que resulta necesario desarrollar un nuevo sistema de asignación de competencias locales que permita desarrollar la autonomía local y aplicar correctamente el binomio subsidiariedad-eficiencia (Círculo de Empresarios, 2007).

\subsection{Agrupación municipal}

Por parte de la Fundación Democracia y Gobierno Local, se elaboró un documento técnico (Jiménez, 2012), en el cual se esbozan ciertas tendencias para la revisión de las administraciones locales. Resulta muy interesante valorar las distintas vías propuestas en el mencionado documento técnico sobre la reforma de la Administración local en las provincias con mayor y menor densidad de municipios, y analizar -a modo de simulación- los efectos que conllevaría la aplicación de propuestas para mejorar la eficiencia en el marco de las administraciones locales. Desde esta óptica, la fusión de ayuntamientos se ha entendido de dos formas muy distintas: en el norte de Europa pretendiendo crear municipios más grandes desde el punto de vista territorial y demográfico (a través de fusiones de pequeños y medianos municipios), y en el sur defendiendo la permanencia de los pequeños municipios ya constituidos (en muchos casos durante los siglos XVI y XVIII). Qué duda cabe, la agrupación municipal supone un claro ahorro para las arcas municipales a pesar de la oposición de movimientos localistas que invocan el carácter de pertenencia y el posible desarraigo de los ciudadanos. la fusión de municipios se ha llevado a cabo en Escandinavia, en el Reino Unido y en algunos Länder de Alemania organizados en dos fases: una voluntaria, y otra obligatoria. Esta iniciativa ha tratado de ser imitada en el sur de Europa con la ley 22/2012 promulgada en Portugal, junto con la iniciativa italiana de reducción de provincias. La fusión forzada de municipios fue implementada durante la posguerra, y también durante la década de 1970 en el Reino Unido, en Suecia y en algunos Länder de Alemania. En nuestro país resulta 
representativo el caso de Madrid, pues entre 1948 y 1954 -también en plena época de crisis económica y social tras la Guerra Civil- acometió la adhesión a esta ciudad de los municipios limítrofes, teniendo como resultado que el término multiplicara por nueve su tamaño original llegando a los $607 \mathrm{~km} 2$, y alcanzando los 2250000 habitantes en 1960. En la actualidad, Madrid es la tercera ciudad europea por población, y la segunda en economía y calidad de vida (según el barómetro Economía del Ayuntamiento de Madrid). En España, el Estado ha de respetar suficientemente la competencia autonómica sobre alteración de términos municipales o sobre organización territorial; esto exige que el enunciado de los criterios sea muy abierto o con amplio poder de excepción para las comunidades autónomas. Qué duda cabe, más cobertura competencial tiene el Estado si en lugar de normas concretas y terminantes, se sirve de normas principales imponiendo un objetivo alcanzable por distintas vías y con diferentes intensidades. No obstante, si bien el Estado puede incentivar la fusión de municipios (mediante medidas económicas o ayudas técnicas), cada fusión concreta ha de acordarse desde cada comunidad autónoma bajo la hipótesis de que el incentivo de las fusiones se concrete en beneficios económicos o subvenciones a los municipios fusionados (de acuerdo con el Tribunal Constitucional), y siempre que deje un margen a las comunidades autónomas para concretar -con mayor detalle- la afectación o destino o, al menos, para desarrollar y completar la regulación de las condiciones de otorgamiento de las ayudas (Sentencia del Tribunal Constitucional n. ${ }^{\circ}$ 13/1992). Excepcionalmente, asegurar la autonomía local a través de recursos financieros suficientes sería competencia exclusiva del Estado como determina el título sobre Hacienda General (artículo 149.1.14. ${ }^{a}$ de la Constitución Española). Por lo tanto, el Estado puede ofrecer financiación de estímulo a municipios que voluntariamente se fusionen o agreguen a otros de mayor escala. El modelo de norma incentivadora sería una previsión expresa de financiación reforzada para todos los municipios, a partir de un determinado segmento de población; o -combinado con esto- una fórmula de financiación específica y favorecedora para los municipios que resulten de la fusión o agregación de otros existentes, siempre que de tales operaciones se alcance un determinado nivel de población. El Banco Central Europeo efectuó unas declaraciones públicas sobre las reformas italianas, en las que defendía que todas aquellas actuaciones que generen economías de escala en los servicios públicos locales deben ser fortalecidas. Para conseguir ese fortalecimiento democrático, el alcalde no debería presidir el Pleno, sino que su presidente habría de ser elegido de entre los concejales. Con objeto de asegurar que el Pleno sea el centro del tablero de la política local (y un fuerte contrapeso al alcalde), deben articularse y ponerse en práctica -activamente- mecanismos institucionales que den apoyo y garanticen el pluralismo de los agentes e interesados en la esfera local, y su interacción con el Pleno (Heilnet, 2010), haciéndolo -además- de manera proporcional en el tiempo en relación al porcentaje de concejales conseguidos por cada partido político. Dicho presidente del Pleno formaría parte -junto con el alcaldede las comisiones territoriales de la comunidad autónoma. A tenor de la legislación vigente, el Estado no puede actuar sobre la planta comarcal de ninguna comunidad; a lo sumo, puede establecer algunos límites negativos a la facultad autonómica de 
configuración de la planta comarcal (tal y como dispone el actual art. ${ }^{\circ} 42.2$ de la Ley Reguladora de Bases de Régimen Local), con el objetivo de garantizar los intereses de los municipios susceptibles de agrupación en una comarca (Sentencia del Tribunal Constitucional n. ${ }^{\circ}$ 214/1989).A los efectos de evitar posibles sentimientos de desarraigo, una propuesta de actuación eficaz supondría que los gobiernos resultantes de la agrupación de municipios (previsiblemente más fuertes al centralizar más poder y administrar una mayor población) sean también gobiernos abiertos, llegando incluso- a establecer que determinadas decisiones sean tomadas en referéndum presencial o utilizando las tecnologías de la información y la comunicación, reforzando así el sentimiento de pertenencia al nuevo municipio, participando más activamente que en la anterior configuración. En países modernos y desarrollados (como Suiza) funcionan sistemas cercanos a la democracia directa, en los que un grupo de ciudadanos puede poner en disputa leyes que hayan sido aprobadas por el parlamento, si previamente llegan a conseguir más de cincuenta mil firmas que estén en contra de dicha ley y dentro de un plazo de cien días.

\section{Intervención de las diputaciones}

A través de esta medida indirecta, se erige la provincia como ente gestor y protector de los municipios de su ámbito territorial. Esta medida ha sido aplicada de forma consensuada entre los municipios y la provincia en los Landkreise (en Alemania). La capacidad inicialmente asignada a las provincias para socorrer a los municipios se ha llevado a cabo en los Megyék (en Hungría), los cuales se han visto desbordados ante la elevada cantidad de solicitudes (debido -esencialmente- a la complicada situación económica).La intervención de la provincia en competencias originarias de los municipios forzaría a cada provincia a un sistema de competencias único, pues la composición y distribución de municipios es heterogénea. En el caso de España, los municipios con población inferior a 1.000 habitantes suman 4.864, lo que supone casi un $60 \%$ del total. Si asistieran a los municipios de menos de 5.000 habitantes, esto supondría 6.800 (casi el 85\% del total de municipios). De aplicar esta política de forma generalizada, las provincias se convertirían en las verdaderas gestoras de las políticas locales en España, dándose la circunstancia de que los ciudadanos habrían elegido a una serie de alcaldes que delegarían varias de sus competencias propias a las Diputaciones (en las que no existe cargo electo alguno). Esta medida se enfrenta a grandes dificultades en su aplicación real, debido a las asimetrías que generaría entre provincias. 


\section{Asociaciones municipales}

La práctica de la asociación de municipios para gestionar determinados servicios es centenaria; según el Ministerio de Administraciones Públicas, la primera mancomunidad data del año 1409 (en el País Vasco) y fue creada para gestionar recursos forestales. Por su lado, las Diputaciones Forales se originaron al consolidarse los Regimientos (reuniones surgidas ante la insuficiencia de las Juntas Generales o su Consejo Real, debido al aumento de la población y sus necesidades -Altuna, 2012-).

Tabla 2. Valores demográficos asociados al número de mancomunidades en España en las provincias con menor y mayor densidad de municipios.

\begin{tabular}{|c|c|c|c|c|}
\hline Provincia & $\begin{array}{c}\text { N. }{ }^{\circ} \text { de } \\
\text { municipios } \\
\end{array}$ & $\begin{array}{l}\text { N. }{ }^{\circ} \text { manco- } \\
\text { munidades } \\
\end{array}$ & $\begin{array}{c}\text { Población } / \mathrm{N}^{\circ} \\
\text { mancomuni-dades }\end{array}$ & $\begin{array}{c}\text { N. }{ }^{\circ} \text { Municipios/ } \\
\text { mancomuni-dades }\end{array}$ \\
\hline Murcia & 45 & 5 & $292.395,80$ & 9 \\
\hline Ciudad Real & 102 & 19 & $27.865,95$ & 5,37 \\
\hline Córdoba & 75 & 10 & $80.510,80$ & 7,5 \\
\hline Albacete & 87 & 16 & $25.105,13$ & 5,44 \\
\hline Cádiz & 44 & 4 & $309.184,75$ & 11 \\
\hline Lugo & 67 & 3 & $117.834,67$ & 22,33 \\
\hline Jaén & 97 & 1 & $670.761,00$ & 97 \\
\hline Asturias & 78 & 19 & $57.070,58$ & 4,11 \\
\hline Sevilla & 105 & 18 & $106.505,39$ & 5,83 \\
\hline Badajoz & 164 & 34 & $20.356,97$ & 4,82 \\
\hline Vizcaya & 112 & 15 & $76.914,93$ & 7,47 \\
\hline Guipúzcoa & 88 & 22 & $32.148,32$ & 4 \\
\hline Barcelona & 311 & 34 & $162.092,56$ & 9,15 \\
\hline Gerona & 221 & 20 & $37.652,30$ & 11,05 \\
\hline La Rioja & 174 & 30 & $10.747,17$ & 5,8 \\
\hline Ávila & 248 & 28 & $6.139,14$ & 8,86 \\
\hline Segovia & 209 & 26 & $6.318,00$ & 8,04 \\
\hline Salamanca & 362 & 31 & $11.407,06$ & 11,68 \\
\hline Tarragona & 183 & 13 & $62.186,15$ & 14,08 \\
\hline Valladolid & 225 & 23 & $23.201,74$ & 9,78 \\
\hline
\end{tabular}

Fuente: Elaboración propia. (Leyenda: Valores mínimos. Valores máximos). 
Aunque aparentemente esta medida mixta tiene los elementos para ofrecer el mejor servicio posible a los ciudadanos, últimamente ha sido necesario el cierre o desmantelamiento de varias mancomunidades. Los ayuntamientos de reducida población se han venido refugiando en esta línea de actuación, visto el considerable aumento del número de mancomunidades en España (actualmente en número superior a mil), mostrando su creciente incapacidad para ofrecer los servicios que dimanan de sus competencias propias (sin entrar en el debate sobre las competencias impropias realmente ejercidas por estos).

La tabla anterior (Tabla 2) muestra cómo las mancomunidades son más frecuentes en provincias con abundancia de municipios pequeños; la formación de mancomunidades o consorcios está directamente dirigida por la comunidad autónoma correspondiente. Como ejemplo destaca Jaén, donde únicamente existe una mancomunidad debido a que la Diputación ha impulsado la creación de consorcios. El cuerpo legal que regula la mancomunidad de municipios se concentra en el Real Decreto Legislativo 781/1986 (por el que se aprueba el Texto Refundido de las Disposiciones Legales vigentes en materia de Régimen Local); en concreto, su art. 35 acota las actividades permitidas a las mancomunidades y establece la formación de su órgano de gobierno. Por otro lado, según la redacción de la Ley 57/2003 (de 16 de diciembre, de medidas para la modernización del gobierno local), en su art. 44 se desarrollan los pasos para su constitución, quedando supeditada al marco normativo de las comunidades autónomas.

Analizando el mencionado Real Decreto, se observan varias limitaciones legales que dificultan la creación de mancomunidades. Como ejemplo encontramos los casos de Cataluña y Aragón, donde se ha promovido una organización comarcal que prohíbe a los Ayuntamientos mancomunarse con municipios de otras comarcas (Riera et al, 2005). En Extremadura se está en proceso de promoción de las comarcas, fomentando la reconversión de las mancomunidades en otras de carácter integral con necesidad de continuidad territorial (o de compartir identidad cultural geográfica o histórica), y no formando parte de otras mancomunidades, al tiempo que son consideradas como embriones de las futuras comarcas. Para tratar de optimizar las posibilidades de éxito de esta manera de mejorar la eficiencia en la Administración pública, las mancomunidades deberían ser gestionadas desde un ámbito externo a los municipios, con objeto de evitar tensiones locales y duplicidades dentro de las entidades locales. De este modo, se trataría de encontrar un equilibrio entre las bondades de las mancomunidades y una amplia representación democrática de los agentes participantes. A modo de resumen de este apartado se concretan -a continuación- las debilidades y fortalezas de las distintas líneas de acción analizadas: 
Tabla 3. Resumen de las fortalezas-debilidades de cada una de las líneas de acción

\begin{tabular}{|c|c|c|c|}
\hline & $\begin{array}{l}\text { Agrupación } \\
\text { municipal }\end{array}$ & Disociación funciones & Asociaciones municipales \\
\hline Fortalezas & $\begin{array}{l}\text { Ahorro } \\
\text { económico. } \\
\text { Resultados } \\
\text { inmediatos. }\end{array}$ & Impide el desarraigo & $\begin{array}{c}\text { Impide desarraigo. } \\
\text { Teóricamente supera el } \\
\text { modelo de mancomunidades. }\end{array}$ \\
\hline Debilidades & $\begin{array}{l}\text { Posible desarraigo. } \\
\text { Necesidad de } \\
\text { vencer la inercia } \\
\text { localista de } \\
\text { determinadas } \\
\text { partes de la } \\
\text { sociedad. }\end{array}$ & $\begin{array}{c}\text { Base legal poco clara. } \\
\text { Ahorro económico no } \\
\text { garantizado. } \\
\text { Generación potencial de } \\
\text { desigualdades entre } \\
\text { provincias. }\end{array}$ & $\begin{array}{l}\text { Resultados graduales. } \\
\text { Poca uniformidad en cesión } \\
\text { de funciones administrativas. } \\
\text { Iniciativa voluntarista. } \\
\text { Poca eficacia de gobierno. } \\
\text { Financieramente débiles. }\end{array}$ \\
\hline
\end{tabular}

Fuente: Elaboración propia.

\section{Particularización de las medidas y propuestas de aplicación del método DEA (Data Envelopment Análisis)}

La Comunidad Autónoma de la Región de Murcia parece haber seguido una estrategia particular, pues es la provincia con menor densidad de municipios (es decir, con los municipios más grandes), dado que en los municipios actualmente existentes se concentran varios núcleos urbanos (pedanías). Estas pedanías han fomentado la progresión dendrítica de la urbanización en la Región de Murcia (Rosa et al., 2012). En primer lugar, si la Región de Murcia optara por fusionar sus municipios de forma sistemática, se podría llegar obtener un número reducido de municipios con una considerable masa crítica y una gran homogeneidad; esta acción recuperaría la configuración original de varias poblaciones de la Región de Murcia, con el claro de ejemplo de los pueblos del Valle de Ricote; todos estos municipios fueron escindidos de la Gobernación de Villanueva de los Infantes por la jurisdicción de primera instancia -en el plazo de un mes- durante el otoño de 1588 (Chacón y Fresneda, 2002). En segundo lugar, y con respecto a la intervención de la diputación, la Comunidad Autónoma de la Región de Murcia -al ser una comunidad uniprovincialcarece de diputación provincial. La provincia de Murcia únicamente tiene 9 municipios con una población inferior a 5000 habitantes (un 20\% frente al $84 \%$ de media en el resto de España). La función de la provincia como agente supervisor de las competencias queda -en esta situación- desvirtuada con respecto a otras provincias, lo cual no significa -en absoluto- que no se deba atender y dar soporte a 
los municipios que lo necesiten, pero -innegablemente- pone en tela de juicio el papel de las diputaciones, a no ser que estos entes sean capaces de adaptarse a las necesidades existentes en cada caso. En tercer y último lugar, cabría hacer referencia a las asociaciones municipales: la morfología de los municipios en la Región de Murcia parece poco propicia -de manera general- para una eficiente implantación de este sistema, ya que los municipios son relativamente grandes, al tiempo que existen enormes diferencias entre los municipios grandes y pequeños dentro de la propia Región (lo que favorecería la generación de tensiones internas entre mancomunidades constituidas por municipios grandes y pequeños).

Partiendo de esta base, parecería del todo recomendable analizar los rendimientos de los municipios desde la prestación de los servicios públicos que ofrecen (alumbrado público, abastecimiento de agua, saneamiento, recogida de residuos, limpieza viaria, etc.). Por su versatilidad, para identificar los municipios más eficientes cabría emplear el método Data Envelopment Analysis (DEA). Para la correcta utilización de este método es necesario conocer los costes (insumos) que tienen los servicios públicos para los ayuntamientos, así como la cantidad de unidades de servicio producido en cada caso (por ejemplo: longitud de calles limpiadas, kilovatios/hora de alumbrado público, número de luminarias, número de contenedores de recogida de residuos, metros cúbicos de agua potable suministrados, etc.). Una vez conocemos cuánto insumo (€) produce cuántas unidades de producción para cada servicio, se puede medir la productividad para poder compararla con municipios similares (en la nomenclatura propia se emplea el acrónimo DMU (Decision Making Units) para definir cada unidad de toma de decisiones (en este caso, cada municipio). La representación tradicional para medir la productividad, consiste en calcular la relación entre la creación de valor agregado -es decir, la producción propiamente dicha- $(Y)$, y el valor de los factores productivos o costes involucrados en el proceso de creación de ese valor $(X)$. Bajo ese planteamiento, las medidas de productividad parciales para cada coste (PP) se pueden definir del siguiente modo:

$$
P P=Y / X i
$$

El citado método DEA es una técnica basada en la programación matemática que, dada una DMU (en este caso, un municipio), nos permitiría identificar aquellas otras variables que nos sirvieran para construir una DMU virtual en virtud de la cual podríamos comparar con la DMU sometida a análisis (municipio). Se trata de una técnica general, que permite trabajar en contextos de múltiples insumos y productos. Tras la medición de la producción, es posible estimar la eficiencia de cada municipio en la prestación de cada servicio (eficiencia técnica); este concepto se puede representar a través de un eje de coordenadas. En las abscisas estaría representada la división entre el primer insumo -o coste- (por ejemplo, el coste de personal) y la cantidad de producto producida (por ejemplo, la superficie de calle que se limpiaría); se representa como eje X1/Y (cf. Figura 2). 
Figura 2. Ejemplo de cálculo de eficiencia según el método DEA

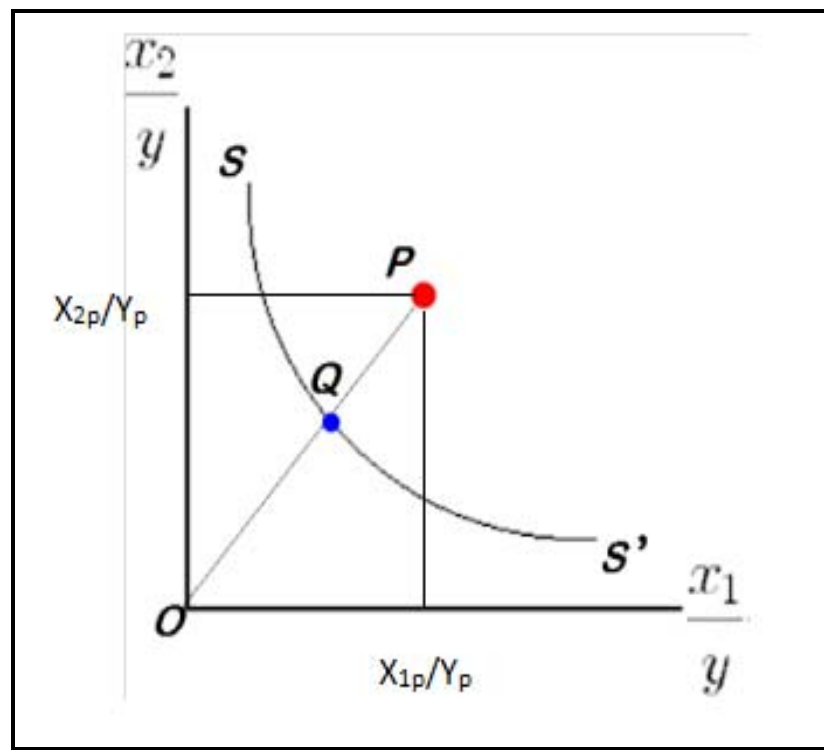

Fuente: Schushny, 2007, p. 12.

En el eje de las ordenadas se situaría el segundo coste (por ejemplo, el coste de maquinaria) o el insumo entre la cantidad de producto producido (por ejemplo, la superficie de calle que se limpia, igual que en el caso anterior); se representa como eje X2/Y (cf. Figura 2). Esta eficiencia técnica se sirve -específicamente- detoda una serie de expresiones (punto $P$, punto $Q$, segmento $Q P$, cociente $Q P / O P$, y eficiencia técnica $-E T-$ ) que pasamos a describir brevemente:

- Punto P: representa la eficiencia técnica del municipio que produce una cantidad $Y$ con los costes $X 1$ y $X 2$; este punto tiene como coordenadas $X 1 p / Y p$ en el eje de las abscisas y $X 2 p / Y p$ en el eje de las ordenadas.

- Punto $Q$ : es la referencia de la máxima eficiencia posible para el municipio representado en el punto $P$.

- Segmento QP: es la reducción de costes que a la que se puede llegar sin dejar de producir una cantidad de producto $Y$.

- Cociente $Q P / O P$ : corresponde a la proporción en la que se puede reducir los costes sin disminuir la cantidad de producción.

- Eficiencia Técnica $(E T=O Q / O P)$ : mide el grado de la eficiencia; cuanto menor sea este segmento, más eficiente se muestra el municipio $P$; si este segmento tuviera longitud cero (0), sería todo lo eficiente que puede llegar a ser. 


\section{Conclusiones}

Una vez analizadas las dos series de provincias españolas con mayor y menor número de municipios por superficie, y habiendo comparado las diversas líneas de actuación previstas para aumentar la eficiencia de las Administraciones locales, la primera conclusión encontrada es que se trata de un ámbito de actuación muy heterogéneo. Dentro de este marco, no parecen viables las soluciones generales y de aplicación directa, haciéndose necesarias medidas específicas que contemplen la identidad y características de cada zona. Tras un análisis del grado de desarrollo/prestación de servicios (eficacia) de la Administración local, se aprecia que trabajar con entidades de escala de nivel intermedio, ofrece las mejores oportunidades debido a su masa crítica y facilidad de gestión. Por esta razón, una de las soluciones más adecuadas parece la orientada a conseguir un tejido de municipios homogéneo y con masa crítica representativa en el ámbito de cada región, siendo necesario -no obstante- potenciar la sensibilización de los usuarios relacionados y su participación. Para hacerlo, los siguientes aspectos habrían demostrado ser esenciales:

- Respetar a todas las partes interesadas, en especial a los ciudadanos; esto es válido tanto para la fase de diseño y desarrollo, como también para su aplicación.

- Adoptar un enfoque positivo al abordar las acciones a emprender.

- Realizar cambios simples prestando atención a los efectos tangibles a corto plazo.

- Focalizar -en la escala humana- la mayor parte del esfuerzo (hasta donde sea posible).

- Cuidar y mantener un enfoque integrado en la formulación de políticas, legislación, asunción de responsabilidades, en la planificación y en el desarrollo económico.

Con la capacidad actual que nos ofrecen las tecnologías de la información y la comunicación, y su gran versatilidad, es factible superar los posibles problemas añadidos por las medidas a tomar para mejorar la eficiencia en la Administración local, independientemente de la medida adoptada. La planificación es, desde la toma de decisiones hasta su implementación, el interfaz clave entre la Administración y los ciudadanos, y será necesaria la inclusión de las nuevas tecnologías a pesar de las dudas y reticencias que puedan surgir en un principio (Vicente, 2011).Como se desprende del punto anterior, para la Región de Murcia la medida de agrupación municipal parece la más indicada; las grandes unidades territoriales han demostrado ser la opción más adecuada para afrontar y solucionar problemas; pero las oportunidades que tienen los ciudadanos de participar en la toma de decisiones, pueden ser percibidas por estos como algo más limitadas. Las soluciones que se propongan e implementen para resolver el inframunicipalismo han de respetar la identidad territorial de los ciudadanos (Nijman, 2000), reconociendo los aspectos 
históricos y culturales asociados a dicha identidad. De esta manera, será posible diferenciar entre la preservación de dicha identidad y la búsqueda de una distribución y gestión del poder en el territorio más eficaz. Debido a la configuración de los municipios en Murcia -que ya tienen un tamaño mediano o grande con respecto a España- se hace posible la aplicación de las medidas en entornos de población medianos. Esta circunstancia favorece la efectividad de las medidas que se pueden adoptar, dando un paso más en el aumento de la eficacia de la Administración local. Por haberse pre-agrupado las pedanías a los núcleos de mayor población (formando un único Ayuntamiento en cada caso), la Región de Murcia está en disposición de realizar la siguiente fase de reordenación territorial en aras de mejorar la eficiencia de sus ayuntamientos. No obstante, debido al conocimiento acumulado, las experiencias de los ejemplos anteriores y la tecnología disponible, se está en disposición de convertir las posibles complicaciones inherentes a la fusión de municipios en vías de solución a los problemas reales que -en la actualidad- apremian a las corporaciones locales. Dada la configuración de la Región de Murcia y su peso relativo en el conjunto de la nación, no parece -en absoluto- descabellado entender que podría convertirse en una comunidad pionera en este sentido, desarrollando medidas para mejorar la eficiencia de la Administración local, y permitiendo que con sus resultados otras comunidades puedan ir adaptándolos progresivamente a sus circunstancias, sin perder de vista que el objetivo es transitar hacia esa necesaria reconversión general de la Administración territorial en España. Por lo tanto, y a modo de cierre final, pareciera del todo razonable entender que aplicando a los municipios el método DEA de forma sistemática, se podrá evaluar el desempeño de estos en la prestación de los servicios públicos en cada provincia. Así, se podrían identificar los municipios más eficientes que -para el resto- servirían de (en palabras del propio autor -Schushny-) benchmark o referencia. Siguiendo esta metodología, se podrán identificar los aspectos sociales, territoriales, funcionales y económicos de los municipios más eficientes, permitiendo a los poderes legislativos (especialmente Administración local y regional) aplicar las políticas adecuadas en cada caso, con objeto de que sus municipios converjan en los parámetros de máxima eficiencia y explotando todo su potencial.

\section{Bibliografía}

Altuna, A. (2012) El origen de las Diputaciones Forales. Navarra. Nabarralde-k argitaratua

Bosquea, J. y García, R. (2000). El uso de los sistemas de Información Geográfica en la planificación territorial. Anales de Geografía de la Universidad Complutense, 20, 49-67. Disponible en

http://revistas.ucm.es/index.php/AGUC/article/view/AGUC0000110049A

Chacón, F. y Fresneda, R. (2002). Los municipios de la Región de Murcia. Origen y análisis de un proceso histórico. Murcia: Asamblea Regional de Murcia.

Círculo de Empresarios (2007). Un nuevo modelo para los ayuntamientos españoles. Madrid: CE. 
Feria, J. y Andújar, A. (2015). Movilidad residencial metropolitana y crisis inmobiliaria. Anales de Geografía de la Universidad Complutense, 35(1), 13-40. Disponible en http://revistas.ucm.es/index.php/AGUC/article/view/48962

Heinelt, H. (2010). Governing Modern Societies: Towards participatory governance.Londres: Routledge.

INE (2012). Instituto Nacional de Estadística. Madrid: INE.

Jiménez, R. (2012). Elementos para un debate sobre la Reforma Institucional de la Planta Local en el Estado Autonómico. Barcelona: Fundación Democracia y Gobierno Local.

Ley 57/2003, de 17 de diciembre, de medidas para la modernización del gobierno local. Boletín Oficial del Estado, n. ${ }^{\circ}$ 301, 1-36. Disponible en

http://www.boe.es/buscar/pdf/2003/BOE-A-2003-23103-consolidado.pdf

Ley 7/1985, de 2 de abril, Reguladora de las Bases del Régimen Local. Boletín Oficial del Estado, n. ${ }^{\circ}$ 80, 1-85, Disponible en

http://www.boe.es/buscar/pdf/1985/BOE-A-1985-5392-consolidado.pdf

Mcfarlane, C. (2010). The comparative city: knowledge, learning, urbanism. International journal of urban and regional research, 34(4), 725-742.

Nijman, J. (2000).The paradigmatic city.Annals of the Association of American Geographers, 90(1), 135-145.

Parejo, L. (2007). Código Administrativo. Códigos La Ley.

Pérez, R. y Gutiérrez, M. E. (2013). Primeras políticas de vivienda en España y su influencia en la evolución de la tipología residencial: el caso de Benalúa (1883-1956). Revista INVI, 28(78), 221-255. Disponible en

http://dx.doi.org/10.4067/S0718-83582013000200007

Real Decreto Legislativo 781/1986, de 18 de abril, por el que se aprueba el Texto Refundido de las Disposiciones Legales vigentes en materia de Régimen Local. Boletín Oficial del Estado, n. ${ }^{\circ}$ 96, 14282-14295. Disponible en

https://www.boe.es/diario_boe/txt.php?id=BOE-A-1986-9865

Reguera, L., Risco, G. (2012). Propuesta para el debate sobre la reforma de la ordenación territorial y administrativa local. Salamanca. Cosital.

Riera, P., Haas, C., Amer Capdevila, C. y Vilaplana, V. (2005). Las mancomunidades en España. Boletín de la Asociación de Geógrafos Españoles, 39, 151-176.

Rodríguez González, R. (2005). Territorio y gobierno local en España. Un planteamiento de reestructuración por fusión como realidad necesaria. Madrid: Civitas

Rodríguez González, R. (2006) "El territorio local en Europa. Reestructuración de su base organizativa y posibilidades para España”. Santiago de Compostela. Xeográfica, Revista de Xeografía, Territorio e Medo Ambiente.

Rodríguez, R. (2009). Reformar la administración territorial: municipios eficientes y viables. Oleiros: Netbiblio

Rosa, J.P. (2012). “Aplicación de los Sistemas de Información Geográfica a la evolución de los usos de suelo en la Huerta de Murcia. Revisión SIOSE 2005-2009”. Proyecto Fin de Carrera de Licenciatura. Ciencias Ambientales. Universidad de Murcia. 
Schushny, A.R., (2007). El método DEA y su aplicación al estudio del sector energético ylas emisiones de CO2 en AméricaLatina y el Caribe, Publicaciones de las Naciones Unidas, Santiago de Chile.

Vicente, S. (2011).e-Planning, an emerging field. Lisboa: Gabinete Técnico ISCSP-UTL.

Páginas web:

El País, http://ccaa.elpais.com/ccaa/2016/04/07/madrid/1460032038_838983.html

\section{Agradecimientos}

Agradecemos la generosidad que nos ha brindado la Dirección General de Administración Local de la Región de Murcia, y también la Dirección General de Política Local del Gobierno de La Rioja, pues gracias a su vocación de servicio público ha sido posible tener acceso toda una serie de datos esenciales para este trabajo. Del mismo modo, es nuestro deseo expresar el reconocimiento a labor científica prestada por los revisores que han sometido a análisis este artículo, destacando su profesionalidad y rigurosidad; en ese sentido, deseamos mostrar nuestro agradecimiento sincero por la ayuda recibida, así como por las interesantes sugerencias facilitadas hasta alcanzar la versión definitiva de este documento. Sin lugar a dudas, constituyen la clave de bóveda para el desarrollo de una revista científica. 